\title{
L'esercizio di foresight per lo sviluppo del territorio
}

\author{
ANGELO DI GREGORIO EMANUELE FABBRI \\ MARIA CRISTINA MORRA*
}

\begin{abstract}
Obiettivo del paper: Presentare una metodologia innovativa di esercizio di foresight che risponda alle esigenze derivanti dai cambiamenti del contesto sociale, tecnologico ed economico.

Metodologia: Abbiamo analizzato un excursus storico all'interno della letteratura classica della metodologia e degli ambiti di applicazione di esercizi di foresight, per evincere quali siano i limiti di tale approccio. Abbiamo poi elaborato un metodologia innovativa e applicato la stessa a un caso pratico nel settore della nautica da diporto.

Risultati: Attraverso un esercizio di foresight si realizzano schemi di sintesi (le Road Map) che prendono in considerazione le risorse e le strategie degli attori locali e diventano un riferimento per gli operatori economici, gli enti di ricerca e le Istituzioni del territorio.

Limiti della ricerca: Non è stato possibile verificare l'adeguatezza degli output dell'esercizio di foresight nel settore della nautica da diporto, in quanto è necessario attendere il termine dei tempi previsionali di sviluppo delle proposte. Si tratta peraltro di limiti connessi alla natura del modello proposto che non è funzionale ad indicare soluzioni, ma a favorire comportamenti di tipo proattivo.

Implicazioni pratiche: La metodologia costituisce una guida operativa dettagliata allo svolgimento di un esercizio di foresight e anche alla rappresentazione dei relativi output attraverso lo strumento delle Road Map.

Originalità del paper: La metodologia presenta sostanziali differenze dai tradizionali esercizi di foresight tecnologici e dalle realizzazioni più recenti volte a privilegiare anche aspetti di tipo sociale.
\end{abstract}

Parole chiave: esercizio di foresight; Road Map; nautica da diporto

Purpose of the paper: To introduce an innovative methodology for foresight analysis and to align this to the needs of the new economic context in relation also to social and technological aspects.

Ordinario di Economia e Gestione delle Imprese - Criet e Università di Milano-Bicocca

e-mail: angelo.digregorio@unimib.it

** Dottore di ricerca - Regione Toscana

e-mail: emanuele.fabbri@ regione.toscana.it

*** Dottorando in Economia Aziendale, Management ed Economia del territorio - Criet e Università di Milano-Bicocca

e-mail: mariacristina.morra@unimib.it 
Methodology: Starting from a literary review on the foresight topics we elaborated an innovative methodology that was applied to a specific study in boating sector.

Findings: Through foresight exercise we elaborated Road Maps which can support effectively economic operators and institutions in setting their strategies and using local resources.

Research limits: Being Road Maps still in progress, it was not possible to verify if the output of our foresight exercise is adequate to the objectives of our research. Such limits are connected to this particular type of methodology which aim is not to find solutions but to stimulate proactive behaviors.

Practical implications: The methodology represents a very detailed guide for decision maker s to develop a foresight exercise and specific Road Maps.

Originality of the paper: The methodology approach we propose for foresight exercise brings a consistent innovation to the traditional technological approach, taking into account social aspect, too.

Key words: foresight management; Road Map; boating sector

\section{II foresight nella letteratura e nella prassi operativa}

Nei tradizionali studi economici - e anche nella prassi operativa - l'esercizio di foresight è una ricerca finalizzata a prevedere le possibili traiettorie tecnologiche e le ricadute produttive per un dato settore, utilizzando il metodo Delphi e prendendo quale riferimento un arco temporale di lungo termine, tipicamente oltre i venti anni (Amsteus, 2008; Calof e Smith, 2012).

L'approccio classico agli esercizi di foresight è stato messo in crisi dall'evoluzione dei mezzi di comunicazione con la conseguente accelerazione dei processi di diffusione delle informazioni, dalla riduzione dei competitive lead time da parte delle imprese e, non ultimo, dalla necessità di estendere il campo di indagine dall'area meramente tecnologica a quella ambientale e sociale.

Il presente studio si propone quindi di innovare ambito di applicazione e metodologia di realizzazione degli studi di foresight, presentando anche una concreta applicazione al settore della nautica da diporto con riferimento ai territori della Toscana, Liguria e Sardegna.

Il lavoro di foresight consiste in una visione proiettata al futuro di differenti scenari evolutivi, con il fine di fornire a chi lo attua un ventaglio informativo vasto in grado di garantire un vantaggio competitivo alle strategie che intende porre in essere (Amsteus, 2008). Le operazioni che compongono l'esercizio di foresight consistono in: valutazione delle implicazioni di decisioni strategiche attuali, monitoraggio e risoluzione di problemi (tecnici, amministrativi o finanziari) prima che si presentino e studio dello scenario normativo.

Il foresight è quindi uno studio utile per segnalare un elevato numero di task rilevanti per la determinazione delle priorità di policy industriale oltre che per la formulazione delle politiche aziendali degli attori industriali nonché la diffusione di informazioni e il coinvolgimento di tutti gli altri pubblici di riferimento. Deve 
intendersi in definitiva quale supporto alle decisioni strategiche di tutti gli attori coinvolti nel settore.

Le origini di questo strumento si ritrovano nella prima metà degli anni '70, anni in cui è emerso in modo inequivocabile il potenziale di un migliore stato informativo sulla competitività industriale, sulla creazione di ricchezza e sulla qualità della vita. L'esercizio di foresight non consiste pertanto nell'identificazione di future innovazioni scientifiche, ma nella predisposizione di infrastrutture necessarie a riconoscere ed esplorare delle opportunità.

Il foresight tecnologico è stato a lungo utilizzato in nazioni quali Giappone e Francia (Urashima et al., 2012), nelle quali è consolidata una tradizione nelle attività di pianificazione, soprattutto di quella associata alle competenze di tipo tecnologico. Negli anni '90 ha occupato ampio spazio anche in altri paesi quali Germania, Stati Uniti e Regno Unito (Georghiou, 1996).

\section{I limiti dell'approccio classico}

I Technology foresight sono stati realizzati dagli anni '70 agli anni '90 utilizzando in via esclusiva il metodo Delphi (Adler e Ziglio, 1996). Come è noto, si tratta di un'articolata metodologia che prevede la selezione preliminare di un panel di esperti, di norma di nazionalità diverse, al quale sottoporre almeno due questionari somministrati in momenti successivi (all'epoca ovviamente tramite posta ordinaria). L'obiettivo era ottenere un feedback "indipendente" ovvero non influenzato dal contradditorio tipico, ad esempio, di un focus group, ma data la complessità dell'esercizio ne conseguiva un tempo di realizzazione variabile da uno a due anni.

$\mathrm{Si}$ intuisce che, proposto in questi termini, il foresight tecnologico è un approccio applicabile ad una realtà in cui le imprese sono guidate da "forti" istituzioni e, soprattutto, in cui i tempi di risposta del mercato sono lenti, molto diversi da quelli odierni caratterizzati da un'elevata velocità di circolazione delle informazioni. Infatti, le indicazioni che nascevano dai precedenti esercizi di foresight tecnologici giapponesi o inglesi erano di tipo "top-down" e non prendevano in considerazione le reali necessità socio-economiche del paese. Inoltre, coprivano un arco previsionale molto lungo, di circa quindici o vent'anni.

L'attuale contesto sociale, tecnologico ed economico mette a disposizione delle istituzioni e delle singole imprese nuovi mezzi di comunicazione che permettono una veloce interazione e diffusione di informazioni e, quindi, portano ad una riduzione notevole del competitive lead time. Di conseguenza, è possibile conoscere i piani strategici e operativi concorrenti quasi in tempo reale.

A partire dagli anni '90 nasce quindi la necessità di collegare lo svolgimento di un esercizio di foresight a caratteristiche che meglio rispondono alle nuove dinamiche competitive e nelle quali le specificità territoriali assumono un peso via, via crescente. 
In questo nuovo filone metodologico si collocano alcuni esercizi di foresight degli anni '90 quali, ad esempio, quelli realizzati da Gran Bretagna, Australia e Nuova Zelanda (Martin e Johnston, 1999). Si riduce l'orizzonte temporale previsionale a circa dieci anni e diventano centrali gli effetti e le opportunità derivanti dalla globalizzazione e dallo sviluppo di Internet. Inoltre, le sfere concettuali in cui si sviluppano gli esercizi di foresight riguardano maggiormente ambiti relativi ad aspetti socio-economici e al miglioramento della qualità della vita. Di conseguenza si adottano metodologie diverse, ricorrendo al metodo Delphi solo in maniera complementare a strumenti quali analisi di settore e focus group con esperti di diversi ambiti professionali, o swot analysis, come nel caso dell'esercizio di foresight eseguito dall'ente FORST per la Nuova Zelanda. In seguito, anche istituzioni nazionali avviano esercizi di foresight improntati a intercettare esigenze di realtà locali, utilizzando metodi con un'accezione più qualitativa e informale.

Nello specifico, si può osservare come il foresight eseguito da Confindustria Emilia-Romagna nel 2006 per il settore agroalimentare abbia ridotto l'orizzonte previsionale a termini di breve periodo: si passa da innovazioni con limiti temporali ventennali a limiti quinquennali ma, soprattutto, non viene adottato il metodo Delphi all'interno della metodologia (Fondazione Faber, 2006). Infatti, il foresight emiliano, dopo una prima fase in cui individua le aree di ricerca entro le quali eseguire l'intero lavoro, procede con interviste personali dirette guidate da un questionario. Questa metodologia permette di conseguire i vantaggi che il contatto diretto con l'intervistato comporta, ossia la possibilità di recepire maggiori informazioni e personalizzare il colloquio in funzione dell'interlocutore. Al termine dell'analisi, gli output del progetto sono rappresentati in matrici di rilevanza, grafici che classificano le tecnologie emergenti (enabler) in funzione delle aree di impatto dei cambiamenti tecnologici (business driver). Infine, le matrici di rilevanza e le istanze che ne emergono sono state utilizzate come base di discussione da sottoporre ai relativi policy maker.

Successivamente, nel 2011 la Regione Toscana ha promosso due esercizi di foresight sulla base di una metodologia comune: il primo riferito al settore biomedicale e il secondo al settore tessile, due comparti di grande rilievo per il territorio toscano. Anche per i due esercizi toscani l'orizzonte previsionale si riduce a tre e cinque anni e la metodologia assume un carattere maggiormente informale. Infatti, dopo un primo studio della situazione attuale del settore di riferimento in Toscana, si è proceduto con sessioni di brainstorming con i relativi esperti al fine di elaborare schede tecnologiche di prodotto e mappe concettuali, nonché di effettuare segmentazioni funzionali del comparto.

Con il supporto di Università e Centri di Ricerca sono state predisposte analisi funzionali, analisi failure e swot delle tecnologie emergenti, in aggiunta ad analisi documentali e dei trend di evoluzione, confluite infine, in Road Map espositive. Le Road Map realizzate dalla Regione Toscana presentano sia aspetti tecnologici, sia gestionali attraverso lo sviluppo di prodotti, processi produttivi, tecnologie e bisogni in funzione del tempo. Come nel caso del foresight emiliano, queste sono esposte in rapporti finali da diffondere tra i principali policy maker del territorio. 
Dalla descrizione di alcuni dei foresight elaborati a livello nazionale, si può evincere come la scala regionale risolva il trade-off tra possesso di massa critica sufficiente e possibilità di realizzare in forma capillare gli interventi dei policy maker. Anche in Giappone, paese noto come fautore e frequente promotore di studi di foresight tecnologici attraverso il metodo Delphi, si sono sviluppate tecniche diverse, interdisciplinari e mission-oriented (Urashima et al., 2012), mentre in Korea l'orizzonte temporale previsionale delle Road Map si riduce da i convenzionali 3-5 anni a 2-3 anni (Seung-Pyo et al., 2013).

Per questa via, l'esercizio di foresight non si sostituisce alle strategie territoriali delle istituzioni e/o delle imprese, ma il suo processo di realizzazione diventa una sorta di "interfaccia" tra il settore pubblico, le imprese e gli altri attori del territorio, diventando sempre di più un contributo ai rispettivi quadri informativo-decisori (Georghiou et al., 2008).

\section{La metodologia proposta}

La metodologia qui esposta rappresenta un ulteriore significativo passo in avanti nella direzione di porre al centro dell'attenzione le esigenze di sviluppo degli attori del territorio, integrando l'analisi competitiva con quella funzionale del settore in esame. In particolare, il modello presentato nel paper è di tipo normativo ed è stato elaborato dagli autori che hanno coordinato sotto il profilo metodologico anche i contributi di terzi citati nel testo. È stato quindi possibile includere in modo esplicito nell'esercizio di foresight anche le competenze presenti nel territorio al fine di valutarne la coerenza con le potenziali evoluzioni tecnologiche e commerciali.

Come illustrato in Fig. 1, la metodologia proposta si articola in sei fasi:

1) analisi desk

2) analisi sul campo,

3) match con gli ambiti funzionali,

4) analisi comportamenti strategici rilevanti,

5) definizione mappe di posizionamento,

6) presentazione delle Road Map.

Fig. 1: Flow chart metodologia

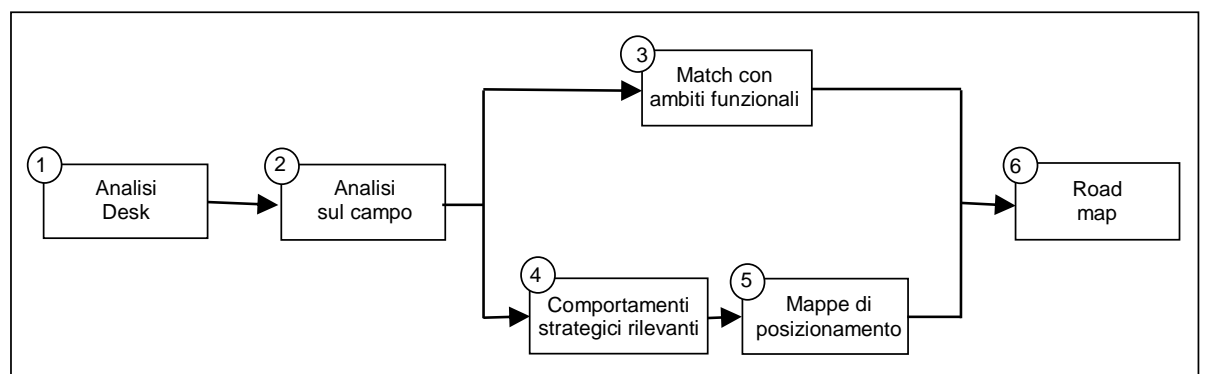

Fonte: Elaborazione propria 
L'analisi desk è funzionale a determinare natura, numerosità e prime caratteristiche, in termini di capacità ed esigenze, degli attori presenti sul territorio con riferimento a un dato settore. La ricerca si concretizza nello studio di pubblicazioni, saggi, brevetti e fonti normative, presenti in banche dati, siti web, e così via. L'obiettivo è costruire un database di contatti di imprese ed eccellenze scientifiche correlate a ciascun ambito funzionale tipico del settore.

Per implementare un esercizio di foresight tecnologico e commerciale, si procede con una serie di interviste agli attori del settore; le interviste sono di tipo strutturato ovvero guidate da un questionario che ne assicuri coerenza e continuità. I partecipanti, attraverso interviste dirette o focus group, individuano temi chiave e driver dei cambiamenti tecnologici del settore, in funzione delle proprie esperienze. Il fine ultimo è cercare di recepire sensazioni e prospettive degli imprenditori, riguardo l'ambiente economico nel quale operano. Oltre agli imprenditori, è opportuno interpellare opinion leader del settore, istituzioni, associazioni e centri di ricerca, per instaurare una collaborazione e una visione interattiva di attori appartenenti a diversi contesti economici e sociali.

Attraverso queste due prime fasi si giunge di fatto a ottenere le informazioni necessarie per un'analisi del settore e, quindi, utile per comprendere il posizionamento competitivo delle imprese. Come illustrato sempre in Figura 1, la metodologia proposta prevede successivamente due percorsi di analisi in parallelo, che condurranno alla determinazione dell'output finale dell'esercizio di foresight, le Road Map.

Da un lato, si effettua un match dei risultati delle fasi precedenti con gli ambiti funzionali tipici del settore. Lo scopo è quello di eseguire un'analisi della situazione tecnologica ed economica che caratterizza il territorio.

Dall'altro lato, si mettono in evidenza i comportamenti strategici rilevanti e si rappresenta il settore attraverso mappe di posizionamento per avere un quadro della situazione competitiva del territorio e tarare l'esercizio di foresight sulle specifiche esigenze delle aziende. Si ricorre quindi, a rappresentazioni di raggruppamenti strategici per riunire le imprese del settore che seguono strategie simili e sottolineare le differenze tra i vari raggruppamenti. Questo studio è funzionale all'individuazione di eventuali/possibili evoluzioni nelle tipiche strategie competitive del settore.

Queste fasi permettono di individuare minacce, opportunità, scelte alternative e figure emergenti da coinvolgere nel processo di formulazione strategico degli attori di un dato territorio: tramite la condivisione di queste informazioni si avvicinano imprese e istituzioni di un territorio permettendo la costituzione di contratti di rete.

Con tutte le informazioni raccolte da analisi desk e interviste si possono ricavare le implicazioni di possibili scenari futuri. Lo strumento utilizzato per rappresentare l'output dell'esercizio di foresight è la Road Map, che consiste in una rappresentazione grafica delle prospettive di sviluppo su un arco temporale abbastanza ampio, come esemplificato in Fig. 2.

Sull'asse delle ordinate sono rappresentate le proposte da attuare, mentre sull'asse delle ascisse l'intervallo temporale, variabile da tre a cinque anni, entro cui sviluppare le suddette attività. Nella parte superiore vengono descritte le competenze 
necessarie alla realizzazione e allo sviluppo delle proposte, per il cui completamento vengono delineate tutte le operazioni necessarie all'interno del grafico. L'ultimo dettaglio è riportato a destra del grafico e serve a raggruppare ed esporre le diverse tipologie della attività proposte.

Fig. 2: Schema di Road Map



Fonte: elaborazione propria

Le Road Map si prestano a diversi utilizzi ma sono essenzialmente riconducibili a favorire una progettualità congiunta per lo sviluppo del territorio e dei suoi attori. Le Road Map infatti, includono in un'unica rappresentazione grafica numerose informazioni eterogenee: eventi esterni che hanno ripercussioni nel settore, prodotti realizzati o necessari, tecnologie complementari e sostitutive, trend tecnologici, sistemi gestionali, competenze e necessità del mercato.

È fondamentale focalizzare l'attenzione su prodotti e processi che hanno implicazioni plausibili e coerenti con il territorio, in questo modo ogni Road Map fornisce ai manager pubblici e privati una cornice di fattori rilevanti per la valutazione e lo sviluppo di efficaci strategie.

\section{II caso Innautic}

La metodologia proposta è stata utilizzata per un esercizio di foresight - il progetto Innautic - realizzato dalle Regioni Toscana, Liguria e Sardegna, con il supporto dell'Agenzia Nazionale per la Diffusione delle Tecnologie per l'Innovazione della presidenza del Consiglio dei Ministri, per il settore della nautica 
da diporto. Nello specifico, non si tratta di un singolo caso ma di un lavoro effettuato su tutta la filiera del settore nautico, coinvolgendo più di una singola impresa. Di conseguenza, è stato possibile toccare molteplici aspetti e diverse esigenze, a partire dal produttore di scafi fino ad arrivare al servizio portuale.

La finalità ultima dell'esercizio di foresight del progetto Innautic è stato quello di rispondere a una reale esigenza dei territori coinvolti, adeguandosi alla velocità di diffusione delle informazioni e dei conseguenti cambiamenti della situazione competitiva che le imprese della nautica da diporto sono chiamate ad affrontare quotidianamente.

Sotto il profilo organizzativo è stato previsto un coordinamento transfrontaliero e, per ogni singola regione, un distinto team di esperti con competenze sia di tipo ingegneristico nella nautica di diporto, sia di tipo economico-aziendale proprio per il fatto di dover realizzare valutazioni non solo di tipo tecnico, ma anche economico.

I lavori sono quindi partiti con l'analisi desk al fine di individuare i potenziali attori da coinvolgere nelle successive fasi. Nel caso specifico, l'analisi desk delle Regioni Liguria, Toscana e Sardegna è stata eseguita rispettivamente da Ires, Dintec e Università di Sassari. I tre istituti, attraverso la metodologia descritta nel paragrafo precedente, hanno mappato imprese ed eccellenze scientifiche presenti sui propri territori, in riferimento a nove ambiti funzionali caratteristici del comparto nautico diportistico $^{1}$. L'output finale di questa fase si concretizza in un elenco di contatti predisposti a essere coinvolti nel progetto e categorizzati in base a core business, ambiti funzionali e provincia di appartenenza. Nella realtà operativa il lavoro svolto in questa fase ha costituito un punto di partenza per gli esperti designati a svolgere le seconda in quanto, per realizzare le interviste hanno selezionato solamente alcune delle aziende proposte e completato l'elenco grazie a propri contatti.

I tre team di esperti hanno quindi effettuato una significativa serie di interviste ai maggiori attori di tutta la filiera della nautica da diporto. Gli intervistati sono stati selezionati, oltre che tra gli imprenditori di riferimento del settore presenti nei territori oggetto di indagine, anche tra opinion leader, istituzioni e associazioni di categoria. Al fine di ottenere un output omogeneo per le tre Regioni e in linea con gli obiettivi del progetto, un coordinatore metodologico ha fornito linee guida per eseguire le interviste e gli esperti hanno declinato di volta in volta le indicazioni in funzione delle caratteristiche e della posizione occupata dall'intervistato all'interno della filiera. Inoltre, al termine di ogni intervista gli esperti erano tenuti a stilare una scheda riassuntiva, in modo tale da rappresentare in maniera lineare e sistematica le principali tematiche e criticità emerse durante il colloquio.

Alla luce dei risultati emersi, le Regioni sono state classificate in relazione alle competenze degli attori del proprio territorio nei diversi ambiti funzionali individuati

1 Gli ambiti funzionali identificati dal progetto Innautic per il settore della nautica da diporto sono: progettazione, sistemi di propulsione, impianti elettrici ed elettroni, uso di nuovi materiali, idrodinamica, interni, servizi per la nautica e il diportismo, mobilità dal porto e attracco al territorio e tecnologie e protocolli di sicurezza per la gestione del traffico merci e persone. 
come prioritari (centri servizi di progettazione, sistemi di propulsione, servizi per il diportismo e, infine, sicurezza del traffico marittimo). Come descritto nel precedente paragrafo, per rappresentare graficamente l'output di queste prime fasi sono state utilizzate delle mappe di posizionamento.

I tre team di lavoro, seguendo le fasi operative descritte in Fig. 1 e per ognuno degli ambiti funzionali individuati, hanno sviluppato distinte Road Map regionali. Successivamente, attraverso l'analisi di quelli che sono gli elementi comuni e complementari tra le singole Road Map regionali è stato possibile predisporre le Road Map transfrontaliere. Infatti, dai rapporti finali regionali sono emerse alcune congruenze tra le esigenze esplicitate dagli operatori di tutta la filiera, nonché alcuni elementi di innovazione che potrebbero essere realizzati in maniera complementare dalle Regioni, in un'ottica di sfruttamento sinergico di competenze, risorse e capacità.

A titolo di esempio, in appendice si presenta una delle Road Map transfrontaliere, relativa all'ambito funzionale dei servizi per il diportismo.

Infine, a partire dal secondo semestre 2012, le Road Map formulate dai tre team di esperti sono state oggetto di specifici confronti in workshop e convegni ${ }^{2}$ promossi nelle Regioni interessate con tutti gli stakeholder al fine di proporre una base sulla quale costruire opportune politiche industriali da parte delle Istituzioni.

\section{Implicazioni manageriali}

La metodologia proposta si differenzia in modo sostanziale dai tradizionali approcci di foresight tecnologico e anche dalle successive realizzazioni volte a privilegiare maggiormente gli aspetti di tipo sociale.

Il metodo qui delineato parte dal presupposto che negli attuali sistemi competitivi il focus non sia più quello di dover identificare delle possibili traiettorie tecnologiche, data la facilità con la quale oggi circolano le informazioni, ma di costruire schemi concettuali, percorsi evolutivi (le Road Map) che possano essere presi come riferimento da operatori economici, enti di ricerca e istituzioni.

L'ipotesi di base è che non sia rilevante solo la ricerca di tendenze tecnologiche e commerciali potenzialmente rilevanti per il settore, ma anche e soprattutto le competenze presenti sul territorio e le strategie in atto da parte dei diversi attori locali. La sfida è rappresentata dal generare sinergie fra gli operatori, da come stimolare comportamenti innovativi superando i limiti naturali del singolo operatore.

2 Gli specifici confronti con gli operatori sono stati:

a) "Progetto Innautic - Forecasting Tecnologico e Governance Territoriale (sottoprogetto C)" - 52 ${ }^{\circ}$ Salone Nautico Internazionale, 11 ottobre 2012, Genova;

b) Innautic-SPC" foresight e nautica in Toscana" - Camera di Commercio, 27 novembre 2012, Livorno

c) "Innautic - Terzarolare nella crisi" - Quartè Sayal, 6 e 7 giugno 2013, Alghero. 
L'approccio di foresight prospettato nel presente lavoro - e realizzato in via sperimentale nel comparto della nautica delle Regioni Toscana, Liguria e Sardegna si propone di fornire un contributo in tale direzione raccogliendo e organizzando le informazioni secondo modalità che, opportunamente condivise, possano dare vita ad una rinnovata progettualità.

I destinatari di tale progettualità si individuano innanzitutto, all'interno di imprenditoria privata e centri di ricerca specializzati, per i quali il fine è quello di predeterminare linee di sviluppo che permettano di focalizzare l'attenzione non solo su aspetti tecnologici di prodotto, ma anche su nuove traiettorie tecnologiche, al fine di stimolare un approccio degli attori del comparto orientato allo sviluppo di una rete di relazioni, servizi e opportunità.

La progettualità inoltre, è finalizzata a favorire la messa a punto di idonee politiche industriali regionali e/o nazionali e quindi, a incoraggiare i policy maker a prendere decisioni per una politica industriale, volta a garantire adeguati sistemi di sostegno allo sviluppo di un determinato territorio.

Sebbene la ricerca presenti limiti relativi alla verificabilità di adeguatezza degli output dell'esercizio di foresight, legati all'attesa del termine dei tempi previsionali di sviluppo delle proposte, questi sono da intendersi agli effetti di lungo periodo delle decisioni degli operatori. Si tratta quindi, di limiti connessi alla natura del modello proposto che non è funzionale a indicare soluzioni, ma a favorire comportamenti di tipo proattivo. Infatti, si tratta di un modello rivolto a definire criteri e metodi idonei a determinare il prevalere delle causalità creativa su quella naturale. In questa direzione sono stati realizzati una serie di incontri con operatori del settore (nota 2).

Vale la pena osservare come la fase di divulgazione dei risultati sia un elemento non secondario dell'approccio proposto, contribuendo in modo determinante ad una migliore comprensione delle caratteristiche ed esigenze degli specifici territori da parte dei soggetti dello sviluppo, imprese e istituzioni.

\section{Appendice 1}

La Road Map, illustrata in modo sintetico in Fig. 3, mostra una serie di proposte - articolate in modifiche tecniche e attività di marketing - che possono costituire i percorsi di sviluppo del turismo nautico nei territori di Sardegna, Toscana e Liguria. 
Fig. 3: Progetto Innautic - Esempio di Road Map nell'ambito funzionale dei servizi per il diportismo

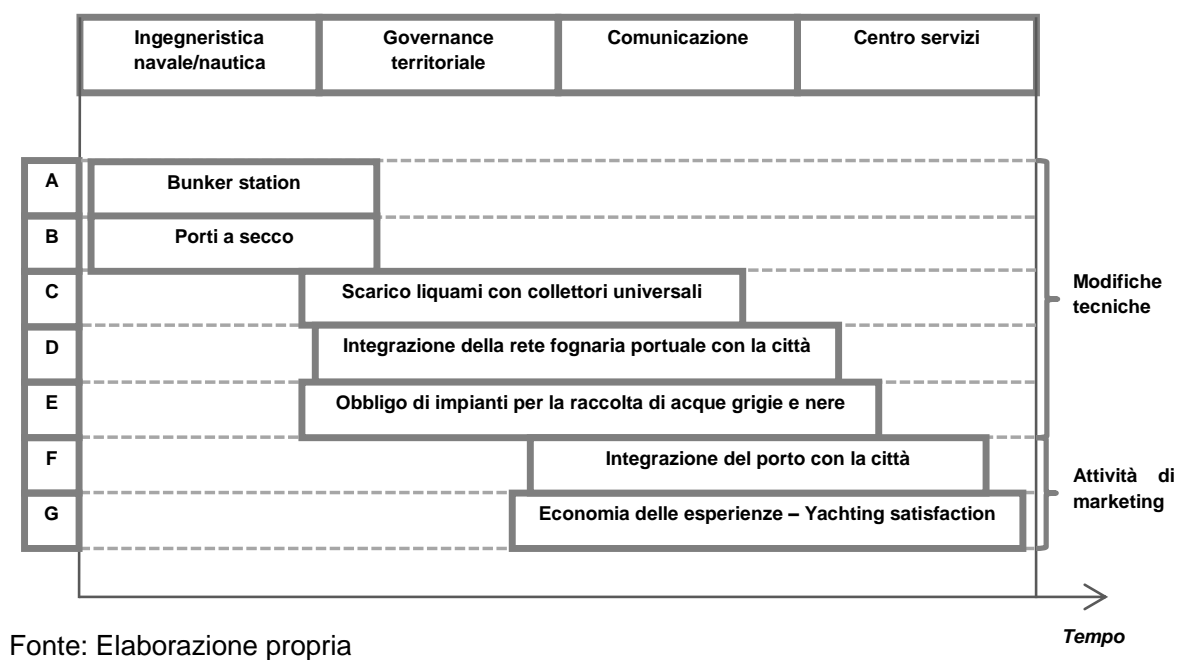

Complessivamente si tratta di sette percorsi di sviluppo ognuno dei quali vede come protagonisti, in momenti diversi, determinati operatori dell'ingegneria navale/nautica, della governance territoriale, delle strutture di comunicazione e di centri servizi. In particolare, i percorsi di sviluppo individuati sono:

A. Bunker station,

B. porti a secco,

C. scarico liquami con collettori universali,

D. integrazione della rete fognaria,

E. obbligo di impianti per la raccolta di acque nere e grigie,

F. integrazione del porto con la città,

G. economia delle esperienze - Yachting satisfaction.

Nel prosieguo del presente lavoro si propone una breve descrizione di ognuno dei suindicati percorsi di sviluppo, senza peraltro entrare nel dettaglio delle diverse fasi che li compongono.

Percorso A. Le bunker station, ossia strutture di fornitura di carburante amovibili, sicuri ed ecologici, rappresentano un sistema innovativo di supporto ai servizi che permettono la liberazione di spazi preziosi da sfruttare come posti barca per la nautica da diporto.

Percorso B. La stessa funzione è espletata dai porti a secco che, oltre a liberare gli spazi per le imbarcazioni, costituiscono un facile sbocco per la piccola nautica, grazie alla riduzione di costi di ormeggio, rimessaggio e manutenzione. Questo strumento per l'aumento dei posti barca è in grado si servire in modo flessibile la piccola nautica, che può adattarsi anche a luoghi al di fuori della conformazione portuale ordinaria. Dislocando i natanti si ottiene maggiore spazio a disposizione 
delle imbarcazioni e della nautica da diporto, evitando i frequenti disagi che sono soliti affrontare i clienti di questo settore.

Percorso $C$. Un altro problema rilevante di questo ambito funzionale è stato specificato nell'assenza di collettori universalmente condivisi per lo scarico liquami. È necessario realizzare un idoneo impianto di bordo, provvisto di terminali di scarico universali che attualmente avviene con ugelli di espulsione dalle dimensioni più disparate, diverse per ogni imbarcazione.

Percorsi D, E, F. Combinando la progettazione di collettori di aspirazione universali con obblighi di impianti per la raccolta di acque nere e grigie in ogni porto e l'annessa integrazione con la rete fognaria cittadina, si otterrebbe un vantaggio competitivo per il turismo nautico nell'area nord tirrenica e ligure. Infine, progettando un collegamento di sbarco direttamente dal posto barca, con tubazioni e collettori di aspirazione dello scarico uguali per ogni tipologia, i diportisti avrebbero la possibilità di scaricare le acque nere e grigie direttamente nel porto, senza problemi di compatibilità tra scarichi.

Percorso G. I precedenti percorsi di tipo tecnico trovano una naturale corrispondenza in una serie di iniziative di marketing e finalizzate a un migliore coinvolgimento, anche emotivo, del diportista. L'integrazione del porto con la città, che promuova più ampie esperienze turistiche caratteristiche del luogo visitato, è attuabile attraverso una gamma di servizi aggiuntivi per quanto attiene sia la fruizione di quelli tipici (quali ad esempio la prenotazione del posto barca, l'assistenza e la manutenzione in loco, ecc.), sia in relazione ai servizi di collegamento con la città, e quindi, con le strutture d'intrattenimento e promozione offerte dal territorio.

\section{Bibliografia}

ADLER M., ZIGLIO E. (1996), Gazing into the Oracle: The Applications of the Delphi Method to Public Policy, Jessica Kingsley Ltd, London.

ALSAN A.M. ATILLA O. (2003), "An integrated view of foresight: integrated foresight management model", Foresight, Emerald Group Publishing Limited, vol. 5, n. 2, pp. 33-45, ISSN: 1463-6689.

AMORIM VARUM C., MELO C., ALVARENGA A., SOEIRO DE CARVALHO P. (2011), "Scenarios and possible futures for hospitality and tourism", Foresight, Emerald Group Publishing Limited, vol. 13, n. 1, pp. 19-35, ISSN: 1463-6689.

AMSTEUS M., (2008), Managerial foresight: concept and measurement, Foresight, Emerald Group Publishing Limited, vol. 10, n. 1, pp. 53-66, ISSN: 1463-6689.

AMSTEUS M. (2011), "Managerial foresight: measurement scale and estimation”, Foresight, Emerald Group Publishing Limited, vol. 13, n. 1, pp. 58-76.

ANTONELLI G., BARSOTTI L. (2011), Rapporto sulla competitività delle imprese toscane nei settori Bio-medicale e Tessile Abbigliamento (Progetto di Foresight Tecnologico)

BONACCORSI A. (coordinatore), (2011), Progetto di Foresight Tecnologico. Settore tessile $e$ abbigliamento, Quinn - Consorzio universitario in ingegneria per la Qualità e l'Innovazione per Regione Toscana. 
BONACCORSI A. (coordinatore), (2011), Progetto di Foresight Tecnologico. Settore biomedicale, Quinn - Consorzio universitario in ingegneria per la Qualità e l'Innovazione per Regione Toscana.

BONACCORSI A. (supervisore scientifico), (2007), I trend tecnologici nel settore della nautica da diporto. Metodologia di monitoraggio, Progetto nazionale di monitoraggio dei trend tecnologici cofinanziato da Unioncamere Nazionale e Dintec, Unioncamere Toscana.

CALOF J., SMITH J.E. (2012), Foresight impacts from around the world: a special issue, Foresight, Emerald Group Publishing Limited, vol. 14, n. 1, pp. 5-14.

DI GREGORIO A., MORRA M.C. (2012), Intervento a convegno "Progetto Innautic Forecasting Tecnologico e Governance Territoriale (sotto-progetto C)", $52^{\circ}$ Salone Nautico Internazionale, Genova, 11 ottobre.

DI GREGORIO A. (2012), Intervento a convegno "Rapporto di Foresight Regionale Progetto INNAUTIC SPC", Innautic-SPC - foresight e nautica in Toscana, Livorno, 27 novembre.

FONDAZIONE FABER (2006), Strategie per la gestione delle alternative tecnologiche del futuro nella filiera agroalimentare, Studio di foresight http://www.confind.emr.it/file.html?_id1=3\&_id2=0000002603.pdf\&_id3=0\&_id4=4 90\&_id5=0\&_sid=ugnkvovuldv

GAVIGAN J.P., SCAPOLO F. (2001), A practical guide to regional foresight, Foren Network (Foresight for Regional Development) - European Commission Research Directorate General.

GEORGHIOU L., (1996), The UK technology foresight programme, Futures, Elsevier Science Ltd, vol. 28, n. 4, pp. 359-377.

GEORGHIOU L., CASSINGENA HARPER J., KEENAN M., MILES I., POPPER R. (2008), The Handbook Of Technology Foresight. Concepts and Practice, Edward Elgar Publishing Inc., Northampton, MA, USA.

HORTON A., (1999), "A simple guide to successful foresight", Foresight, Camford Publishing Ltd, vol. 1, n. 1, pp. 5-9, ISSN: 1463-6689.

JUNG HOON LEE A., PHAAL R., SANG-HO L. (2013), An integrated service-devicetechnology roadmap for smart city development, Technological Forecasting \& Social Change, Elsevier Inc., vol. 80, n. 2, pp. 286-306, DOI: 10.1016/j.techfore.2012.09.020

MARTIN BEN R., JOHNSTON R. (1999), Technology foresight for wiring up the national innovation System, Technological forecasting and social change, Elsevier Science Inc., vol. 60 , n. 1, pp. 37-54.

OKOLI C., PAWLOWSKI S.D. (2004), The Delphi method as a research tool: an example, design considerations and applications, Information and management, Elsevier Science Ltd, vol. 42, n. 1, pp. 15-29.

PHAAL R., FARRUKH C.J.P., PROBERT D.R. (2004), Technology roadmapping - A planning framework for evolution and revolution, Technological Forecasting \& Social Change, Elsevier Inc., vol. 71, n. 1-2, pp. 5-26.

PORTER M.E. (1981), La strategia competitiva. Analisi per le decisioni, Editrice Compositori, Bologna.

SCIENCE AND TECHNOLOGY FORESIGHT CENTER NATIONAL INSTITUTE OF SCIENCE AND TECHNOLOGY POLICY (2010), "The 9th Science and Technology Foresight. Contribution of Science and Technology to Future Society", Capability of Local Regions for the Green Innovation, report n. 142. 
SEUNG-PYO J., JU HWAN S., JONG-KU S., (2013), A study of the SME Technology Roadmapping Program to strengthen the R\&D planning capability of Korean SMEs, Technological Forecasting \& Social Change, Elsevier Inc.,vol. 80, n. 5, pp. 1002-1014.

SLAUGHTER R.A., (1996), Foresight Beyond Strategy: Social Initiatives by Business and Government, Long Range Planning, Elsevier Science Ltd, vol. 29, n. 2, pp. 156-163.

TOLA A., (2013), Intervento a convegno "Introduzione e presentazione della ricerca per la definizione di modelli innovativi da applicare per lo sviluppo economico e tecnologico nel settore della nautica”, Innautic - Terzarolare nella crisi, Alghero, $6 \mathrm{e}$ 7 giugno.

UGHETTO E., (2007), "Foresight as a triple helix of industry, university, and government relations", Foresight, Emerald Group Publishing Limited, vol. 9, n. 5, pp. 14-22, ISSN: 1463-6689.

URASHIMA K., YOKOO Y., NAGANO H., (2012), S\&T policy and foresight investigation impact in Japan, NISTEP, Foresight, Emerald Group Publishing Limited, vol. 14, n. 1, pp. $15-25$.

YASUNAGA Y., WATANABE M., KORENAGA M., (2009), Application of technology roadmaps to governmental innovation policy for promoting technology convergence, Technological Forecasting \& Social Change, Elsevier Inc.,vol. 76, pp. 61-79. 